University of Nebraska - Lincoln

DigitalCommons@University of Nebraska-Lincoln

20th \& 21st Century French and Francophone Modern Languages and Literatures, Department Studies International Colloquium

$4-2020$

\title{
Sylvain Tesson : La Consolation de la forêt
}

Sylvie Blum-Reid

Follow this and additional works at: https://digitalcommons.unl.edu/ffsc2020

Part of the Comparative Literature Commons, French and Francophone Literature Commons, and the Other French and Francophone Language and Literature Commons

This Presentation is brought to you for free and open access by the Modern Languages and Literatures, Department of at DigitalCommons@University of Nebraska - Lincoln. It has been accepted for inclusion in 20th \& 21st Century French and Francophone Studies International Colloquium by an authorized administrator of DigitalCommons@University of Nebraska - Lincoln. 


\section{Sylvain Tesson : La Consolation de la forêt}

«Je vous écris du bout du monde »(Chris Marker) 1

Sylvain Tesson est né sous les constellations du blaireau et de la forêt.2 Cet écrivain-voyageur, géographe de formation, a passé six mois dans une cabane en Sibérie, à la suite desquels il a publié son journal Dans les Forêts de Sibérie traduit en anglais par The Consolation of the Forest en 2011 (prix Médicis 2011). En plus de ses écrits, il est l'auteur de plusieurs documentaires filmés Aral: Chronique d'une mer asséchée, 2002 (Aral, the Sea than Ran dry, dir. Nicolas Millet, 42 min. , CNC),3 qui dénonce la catastrophe écologique de la mer d'Aral, asséchée depuis les années 60, en raison d'une surproduction de coton, et Seul, 180 jours sur le Bä̈kal /Alone, 180 days on Lake Baïkal (2011 co-réalisé avec Florence Tran).4 Il a reçu le prix Renaudot pour la Panthère des neiges (2019), son récit au Tibet.

Je m'intéresse au parcours de Tesson dans différentes régions du monde ainsi qu'en France, comme thérapie nécessaire à son équilibre mental ainsi que son implication dans le système écologique de notre planète. Il va de soi que si tout voyage constitue sa thérapie, la forêt en fait partie. Les questions qui ont guidé ma relecture de Dans les forêts de Sibérie dans le but de notre conférence Parler la Terre, sont les suivantes: Quelle est sa vision de la terre et du système écologique, qui s'inscrit au coeur de ses écrits ? Si l'on fouille un peu dans le roman familial de la famille Tesson, on débouche sur le mythe de la cabane et de la forêt tout en sachant que Tesson et sa famille sont virulemment anti-psychanalyse.5 Il est difficile d'expliquer cet état de non-repos permanent de Tesson, que l'on nomme aussi agitation ou fébrilité. Il se démarque par son désir de vivre loin de notre époque et du style de vie 'moderne', avec tout ce qu'elle comporte : les médias sociaux, ses addictions au téléphone portable, le haut débit, et ses obsessions avec les 'hauts taux' (boursiers et autres), les évaluations, les fonctions 'j'aime' sur Facebook, ainsi que l'édification 

constante des célébrités. Tesson se targue 'd'être de tous les réseaux asociaux'. 6 Pour compliquer les choses, Dans les Forêts de Sibérie célèbre l'érémitisme, le mode de vie des ermites, s'inscrivant contre le voyage ; ceci le guide dans sa quête d'un havre, très loin de la France dans sa région de prédilection : l'Eurasie. 7 «C'est là que je situe pour l'instant mon domaine de prédilection entre le Pacifique et l'Atlantique» (Petit Traité sur l'immensité du monde 2005 :17). Dans les années 90, il traverse l'Himalaya dans une randonnée de 5000 km parcourant les plaines de l'Asie centrale à cheval, et le monde à bicyclette. Ce mouvement constant peut ressembler à une malédiction pour reprendre l'expression de l'écrivain anglais Bruce Chatwin (qui était lui aussi en mouvement). L'expérience sibérienne trouve son cadre dans le voyage immobile, à la base d'une transformation personnelle, et dans l'autobiographie et l'écrit sur la nature. Tesson, le nomade, l'errant, le trappeur, le 'wanderer', le rebelle, le praticien de l'art de la fugue, et de l'appât, échappant à tout réseau, marche à contre-courant, contre l'idée de 'se ranger (le mot passe mieux en anglais : settling down) c'est à dire former une famille et avoir un emploi sédentaire. Sa famille - le clan des Tesson - accepte et respecte son choix. Son père, Philippe Tesson, journaliste de droite, connu pour ses chroniques littéraires et surtout théâtrales, admire son fils, un 'anti-moderne', horsnormes, branché, sauvage et vivant dans l'excès:

"Sylvain a besoin de partir loin dans la solitude et l'excès. Il lui faut quitter une société trop étroite pour lui."8

Dans une lettre-hommage publiée dans le Figaro,9 Philippe Tesson, le père, explique que Sylvain -enfant avait l'habitude de dormir dans les arbres, une confession aux échos durassiens en rapport au texte hybride Des journées entières dans les arbres.

L'expérience sibérienne est toute à la gloire du sauvage. Le fils admet qu'il pratiquait la montée et la descente des montagnes mais que jusqu'alors il n'avait jamais pensé à les regarder (Forêts, 114). Sa réponse à l'appel des forêts' provient de l'énergie vitale maladive dont il a hérité de sa famille.10 Né en 1972, Tesson s’est promis de vivre sur le Baïkal avant ses quarante ans. Il en a 37 quand il entreprend sa quête introspective de six mois dans une isba au Baïkal. Un journal de 
FFSC 2020 conference. "Parler la terre" Sylvie Blum-Reid- U. Florida bord s'ensuit dessinant le lac, long de 531 miles, et de 50 miles de largeur. C'est le plus vaste réservoir d'eau naturel au monde. Le Baïkal est considéré comme une mer par les habitants, les Bouriates/ Mongols : Le Dalaï Nor. Un endroit déclaré comme réserve biosphère par l'Unesco en 1996, « le plus grand terrain vague du monde » selon Chris Marker dans son envolée vers la Sibérie (Lettre de Sibérie, 1958). Tesson emprunte au vocabulaire religieux et animalier pour sa retraite. Bien qu'élevé dans une école de Jésuites, il n’est pas 'religieux' au sens étroit du termeil se considère tel un ermite de la forêt comme ses voisins du lac (Forêts 148), ses affinités sont intimement liées à son prénom Sylvain ;11 proche aussi du blaireau, d’où provient son nom familial Tesson (en vieux français, 'taxos'), un animal à l'affut et à la traque. 12 Selon Buffon : « Le blaireau est un animal paresseux, défiant, solitaire, qui se retire dans les lieux les plus écartés, dans les bois les plus sombres, et qui s'y creuse une demeure souterraine. »13 Tesson s'intéresse aux animaux et aux forêts.

Après de longues heures de camionnettes et l'assistance d'amis, le voyageur arrive le 11 février 2010, avec pour tout bagage des provisions et des outils pour six mois : vodka, pâtes, et sauce Tabasco fournissent le plus gros. Sans oublier une caisse de livres sélectionnés pour ce séjour dans l'anticipation de vivre dans le silence de sa bibliothèque. « Les livres sont plus secourables que la psychanalyse. Ils disent tout, mieux que la vie. Dans une cabane, mêlés à la solitude, ils forment un cocktail lyrique parfait» (Forêts, 247). Sa sélection assez éclectique comporte du Bachelard, Tournier, Thoreau, Mishima, Whitman, Nietzche, Jules Vernes et quelques 'polars'. En décryptage, nous constatons sa prédilection pour la philosophie surtout transcendentaliste de Thoreau et Whitman, ainsi que la poésie, et la science-fiction. Les récits de voyage, et ou récits de naufragés abondent. Il se construit une petite étagère pour ses 'icônes'. Parmi la liste de ses raisons à ce séjour sibérien fournie en milieu de récit, j’en relève quelques-unes qui se distinguent, dans le texte, entourées d'un carton :

J'étais trop bavard 


\begin{tabular}{|l|}
\hline Je voulais du silence \\
\hline Trop de courrier en retard et trop de gens à voir ... \\
\hline Par lassitude d'avoir à faire les courses \\
\hline Pour pouvoir hurler et vivre nu \\
\hline Par détestation du téléphone \\
\hline Et du bruit des moteurs (Forêts, 118) \\
\hline
\end{tabular}

Il fuit toute activité associée aux bruits parisiens (Forêts, 128). Tesson entend simplifier sa vie, la réduire à l'essentiel, et être seul avec lui-même. Pour ce faire, il aménage sa 'cabane', perce une ouverture devant le lac, une fenêtre par laquelle il poursuivra à loisir sa contemplation, ses écrits et lectures. Il se trouve à trois jours de marche d'avec ses plus proches voisins. Il leur rendra visite glissant sur le lac ou les montagnes. De plus, certains visiteurs (des militaires russes) se rendent chez lui, de façon inopportune, et sans frapper. Deux chiots lui seront confiés et qui feront son bonheur dans sa solitude. Il s'apercevra qu'il peut freiner le temps, quelque chose qu'il avait déjà noté lors de ses marches :

La journée s'étire. A Paris, je ne m'étais jamais trop penché sur mes états intérieurs. Je ne trouvais pas la vie faite pour tenir les relevés sismographiques de l'âme. Ici, dans le silence aveugle, j'ai le temps de percevoir les nuances de ma tectonique propre. Une question se pose à l'ermite. Peut-on se supporter soi-même? (Forêts, 54)

Cette dernière question sera réitérée plus loin : “me supporterai-je moi-même?” (Forêts, 111).

Le côté périlleux de ses aventures n'échappe pas au lecteur, commentant en final sur la cabane comme le lieu du pas de côté (Forêts, 267). Quelques années suivant l'expérience sibérienne, et quelques mois après la mort de sa mère d'un cancer en 2014, Sylvain Tesson, grand amateur de varappe, grimpeur chevronné, tombe accidentellement d'un toit dans les Alpes. Il a survécu à quatre mois de coma, métamorphosé mais en gueule cassée (et dos cassé). Cet accident ne l'a pas 

interdites et qu'il souffre de graves séquelles.

\section{Ecologie}

Il va sans dire que l'approche de Tesson est fondamentalement ancrée dans la pensée écologique de la défense de l'environnement, même si sa vision et démarche sont attaquées par certains car elle peut paraître dérangeante. L'écologie ou l'éco-philosophie portant sur la quantité d'empreinte carbonique qu'il faut laisser dans la nature guide ses gestes et dépenses. Sur la même longueur d'ondes que certains géographes environnementaux, Tesson a volontairement quitté les villes surpeuplées et les sphères urbaines, et les habitudes sociales ou L'Habitus de Pierre Bourdieu. 14 Il s'insurge contre l'état du monde, et la relation entre l'être humain et la nature, contre 'L'aménagement du territoire' ou 'l'avancée de l'asphalte' dans plusieurs œuvres, dont Petit traité sur l'immensité du monde (2005, livre antérieur à l'épisode Sibérique)15. Le monde est en péril, et l'équilibre de la planète est menacé. Tesson n'est pas un 'révolutionnaire' ou un 'activiste', mais un poète métaphysicien. Après tout, il vient chercher refuge dans la forêt boréale, loin 'du monde' occidental, signifiant cette même forêt comme nécessaire à sa survie, et pour citer ici Halia Koo, il est marqué par 'la doxa écologique.' ${ }_{16}$ Près du Baïkal, la vie se réduit à des gestes simples et vitaux. Le temps se passe dans la contemplation, et les petits plaisirs, ainsi que le travail physique tel que couper des arbres pour le feu, briser la glace pour utiliser de l'eau, et marcher trois jours pour voir les amis. La plupart des résidents réfractaires au régime Moscovite, partagent ce désir de vivre loin des bruits des villes. Toute communication avec le monde extérieur s'atténue.

Le séjour de Tesson en Sibérie le réconcilie avec le monde ou 'le grand dehors' (de l'écrivainvoyageur Michel Lebris). Il en ressortira plus serein.

Tôt dans son journal, Tesson rejette tout support visuel prétendant qu'il est incapable de prendre la moindre photographie. Cependant, nous savons qu'il a eu recours à une caméra pour enregistrer certains moments dans la taïga, aboutissant à un documentaire savamment monté de 50 minutes 

les effets du film, une nouvelle isba calquée sur l'ancien modèle fut reconstruite dans une autre partie du lac. Tesson revint donc en Sibérie, en tant que consultant et passa même quelque temps dans la nouvelle Isba. (https://www.youtube.com/watch?v=-Q7dmocLZGc)

Chris Marker demeure la référence incontournable pour tout film d'essai sur l'endroit : Lettre de Sibérie (1958, 62 mn.). Ce film fait l'éloge de la Sibérie, et une critique à peine voilée du système communiste. Pareillement, Tesson s'inspire du film de Kurosawa avec le merveilleux Dersou Ouzala (1975).18 Dans celui-ci, il y retrace le parcours d'un vieux trappeur autochtone, un 'homme des forêts' qui rencontre un officier de l'armée impériale russe lors d'une mission dans la contrée. Dersou est un animiste, qui voit tout animal ou plante à l'égal de l'homme, et ne supporte pas de vivre enfermé entre quatre murs. Chaque récit, soit de Tesson, soit de Marker ou de Kurosawa (adapté d'Arseniev) contient des éléments romantiques et extraordinaires. 19 La région était perçue comme un lointain désert balayé par les vents où les opposants au régime Soviétique étaient envoyés dans les Goulags. Toujours la forêt s'y profilait. Elle pouvait être plaisante et accueillante ou parfois dangereuse. Le pays est pour Marker celui de 'l'enfance' relié à ses lectures. La population autochtone a été décimée, très peu y vivent encore ainsi que témoignent Werner Herzog et Dmitri Vasyukov dans leur film: Happy People: A year in the Taïga (2010) qui suit un groupe de trappeurs dans leur hivernage, loin du village de Bakhta, et qui décrivent la 'beauté de l'espace, du froid et du silence.' 20 Le commentaire de Marker résonne encore, ““'Je vous écris d'un pays lointain.” Ce film, comme le texte de Tesson, plus que sur la réalité historique du lieu, constitue une ode lyrique à l'espace et un constat sur notre état du monde.21

1 Chris Marker. “Lettre de Sibérie » Argos Films, 1958. Initialement intitulé Baïkal. 2 Si elles existaient évidemment ! 
3 http://www.film-documentaire.fr/4DACTION/w_fiche_film/11900_1

4https://www.youtube.com/watch?v=JlLaWBXfQxU [with English subtitles]

5 For this verification, check article in JDD 2018.

6 https://www.letemps.ch/culture/sylvain-tesson-suis-reseaux-asociaux

7 Consolations of the Forest: Alone in a Cabin on the Siberian Taïga remporte le prix Dolman Travel Book, et le prix Médicis, spécial Essai 2011

8 "Les Tesson, un clan Romanesque et anti-conformiste," Europe 1, le Journal du Dimanche, 8 mars 2018. https://www.lejdd.fr/culture/les-tesson-un-clan-romanesque-et-anticonformiste3591176

9 Le Figaro, 8 avril 2018.

10 Marie-Laure Delorme. "Sylvain Tesson: Au bout de soi" Europe 1- Le Journal du dimanche. 18 sept. 2011.

11 'L'ermite des taïgas se tient aux antipodes de ces renoncements. Les mystiques cherchaient à disparaître au monde. Le forestier veut se réconcilier avec lui.....ils voulaient l'éternité, il traque l'exaucement. Ils espéraient mourir, il aspire à jouir. Ils haïssaient leur corps, il aiguise ses sens...(148)

12 « Sylvain Tesson. La panthère des neiges. » La maison de la poésie. La scène littéraire.. Télévision. 6 nov. 2019 https://youtu.be/tvwmmrC9RVA

13 «Buffon », Dictionnaires Le Robert - Le Grand Robert de la langue française.2017

14 Habitus: Manière d'être d'un individu, liée à un groupe social et se manifestant dans son apparence physique (vêtements, maintien...).

15 Tesson a compilé des notes parues dans la revue Grand Reportage pour Le Petit Traité.

16 Halia Koo, «Une esthétique de l'esquive : stratégie de résistance et de retrait dans l'espace sylvestre de Sylvain Tesson » Voix Plurielles (16.2) 2019.

17 Sylvain Tesson et Florence Tran. Six mois de cabane au Baïkal (film, 2010).

https://www.dailymotion.com/video/xqgmd6

https://www.youtube.com/watch?v=-Q7dmocLZGc

18 Akira Kurosawa (dir.), Dersou Ouzala, (film Russo-Japonais, 1975) 2h.41mn. Ce film est adapté d'une trilogie autobiographique de Vladimar Arseniev Dersou Ouzala (1921).

19 Lettre de Sibérie (1957, 62 minutes). Cinematography Sacha Vierny, Production Anatole Dauman- Argos Films. He was commissioned to make the film by Anatole Dauman.

20 Je tiens à remercier mon collègue en russe James 'Frank' Goodwin pour m'avoir indiqué ce film et me l'avoir prêté. 
21 Marker évoquant lui -les voyages d’Henri Michaux. 